\title{
Impact Based on Quarantine Psychology: Analysis of left and right brain hemispheres
}

\author{
Abhinanda Sar ${ }^{1}$, Jaspreet Kaur ${ }^{2}$, Manoj Kumar Jindal ${ }^{3 *}$ and Kauleshwar Prasad ${ }^{4}$ \\ Affiliation and address of the author(s): ${ }^{1,2,4}$ Department of Computer Science and Engineering, \\ Bhilai Institute of Technology, Durg, Chhattisgarh, India, 491001; ${ }^{3}$ Department of Chemistry, \\ Bhilai Institute of Technology, Durg, Chhattisgarh, India, 491001. \\ E-mail address of authors: abhinandasar11@gmail.com ${ }^{1}$, jaspreetahden24@gmail.com ${ }^{2}$, \\ manojjindal1989@gmail.com ${ }^{3}$ and kauleshwar.prasad@bitdurg.ac.in ${ }^{4}$ \\ E-mail address of Corresponding author: manojjindal1989@gmail.com*
}

\section{Abstract}

Brain, the most complex object known in the universe, uses few watts of power. To mimic it a nuclear power plant will be required and this power house controls the human body, single handedly. Surprisingly, "On the left side, nothing is right and on the right side there is nothing left". Typically, there are two lateral halves of the brain: Left hemisphere and right hemisphere working distinctly. Left hemisphere is inclined towards logic; Right hemisphere is the root cause of imagination added with critical thinking. In situations like the current pandemic, COVID-19, it is the right half which tends to dominate the processing. This gives birth to mental stress, anxiety thus, aggravating the existing medical condition. Considering this pattern, a survey was conducted in Durg district of Chhattisgarh, which is one of the most hard hit epicentre of the COVID-19 second wave in India. According to this survey, it was revealed that largely women of all age groups (10-25, 26-40, 40 above) were right brained i.e. dominance of right over left hemisphere. Being more imaginative and creative thinkers they are more likely to suffer from mental issues than males. The aim of this research is to improve the mental wellbeing of the citizens in such threatening conditions. To prevent this situation awareness is a must and some stress relieving games have also been created.

Keywords: Mental Health; Machine Learning; Modern Imaging Techniques; Psychogenic fever; rheumatic; Classifier.

\section{Highlights:}

- On studying different age groups, Females are found to be more right brained.

- Mostly females are found to be rheumatic in nature and prone towards depression.

- Males are more Left brained tending to be more logical and calculative in nature.

- Women are most likely to develop a psychogenic fever, when in stress.

- Research has been focused on the age group(10-25), the future generation of India. 


\section{Introduction}

This survey revealed the prominence of the right brain among humans in situations of despair. The COVID-19 pandemic is a significant threat to humanity. The pandemic is putting excessive pressure on our healthcare and economic systems. Further COVID-19 threatens social connections among humans.

\subsection{Brain Hemisphere}

Typically, there are two lateral halves of the brain: Left hemisphere and Right hemisphere working distinctly. It cannot be obscured that both the hemispheres work together to function as a brain but it is scientifically proven fact that each hemisphere has unique functions to be performed.

\subsection{Difference between the two hemispheres}

Out of the two cerebral hemispheres, the dominance of the right hemisphere can be correlated to mental disturbance. People with a right-dominant brain are believed to be more creative, emotional, and intuitive. The general sense of space is dealt by the right hemisphere, whereas the objects in specific locations are dealt by the Left hemisphere.

\subsection{Correlation of Right Hemisphere and mental illness}

Right hemisphere allows to organize data sufficiently in a mono semantic way and it replaces the reality that exists with factitious information.

However, the right hemisphere was more specialized for perception. Some were dubious, about the correlative and symbiotic nature of the two sides of the brain in maintaining psychological equilibrium [1].

Importantly, individuals who are characterized by a creative brain-style could experience harsh effects due to COVID-19. More importantly a person who thinks more deeply ahead of the real world phases threat to their mental and physical health. Some experimental clues lead to the fact that creative thinking is associated with nature to focus on health problems. For example, it is found that individuals with an imaginative thought style are more likely to incur health issues into their own memory, and this intensifies their sense of unguarded existence to illness and anxiety [2]. Therefore, individuals with an abstract brain style experience a greater need for social interaction however supporting social friction and negative views from others.

\subsection{Need of techniques}

The main purpose of this paper is to discuss the relationship between mental issues and hemispheres of the brain. In current scenarios there are multiple tests and online resources which help in determining dominance of one's brain .However, no such relationship has been drawn between the dominance and cure of mental issues. Generally mental wellness is always linked with a social stigma which prohibits people from exploring the possible cure. Until a person discovers of him/her to be suffering from severe mental illness and no steps are taken in the initial level leading to higher degree of deterioration.

Even Though the threat to human interaction is virtually universal, the specific mechanisms by which COVID-19 affects our mental wellbeing are versatile and modifiable across individuals. To support this effort, we advance hypotheses on how personalized brain profiles act in different hemispheres of the brain. The functionalities of Brain are shown in Fig. 1. The research on Hemispheres of the brain could enhance the examination related to these topics. People all over the world are gradually being encouraged or forced to stay at homes, separated from friends, family, and coworkers; except for essential activities. While these techniques are currently necessary to mollify the effects from public health view, this level of quarantine is inconsistent for human behavior $[1,3-4]$. 


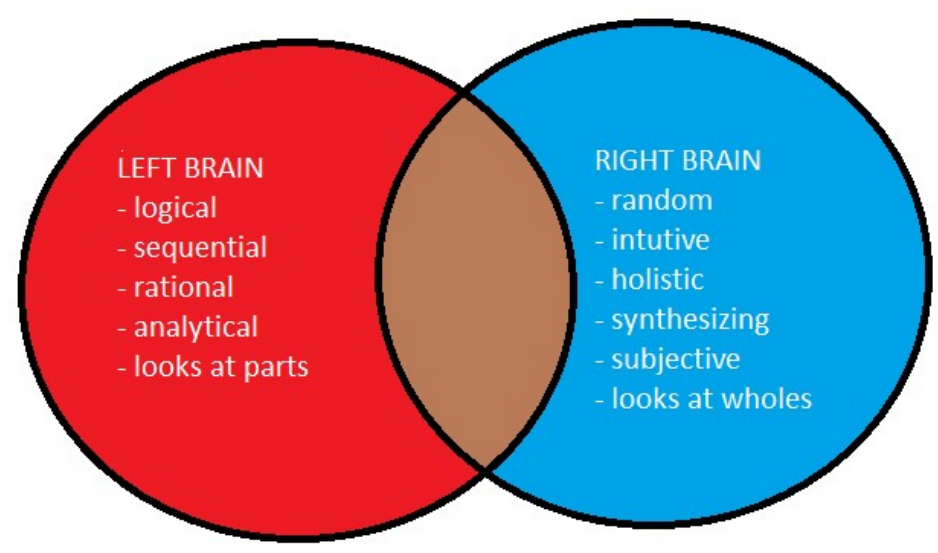

Fig. 1. Functionalities of Brain, when split.

\section{2. $\quad$ Related Works}

In the Nineteen Sixties, initial observations exploded each into the scientific literature and within the public sphere once some spectacular and shuddery experiments were performed [5]. It absolutely was throughout this decade that researchers were experimenting to search out ways to treat severe brain disease.

There was a category of patients whose seizures could not be treated by psychotherapy or psychotherapeutic drugs. These people would have frequent and exhausting and weakening seizures which was no way to live. Thus Gazzaniga [6], Sperry and other scientists [7, 8] explored further treatments.

\subsection{Split Brain Experiment}

One treatment was to sever a part of the brain that connected the right and left hemispheres. These studies are referred to as "Split-Brain Experiments". The tract is an element of the body that connects the two sides of a healthy brain.

In these surgeries, surgeons merely cut the tract (The hemispheres communicate with one another through a thick band of 200-250 million nerve fibers), turning the brain that was once single in operation basically into 2 separate ones. The complete prospect could have given others the sneaks, however the surgeons had some success in treating brain disorder, thence some surgeries were performed [6]. It was from these studies that scientists became conscious of a number of the regional variations of gifts within the functioning of the brain.

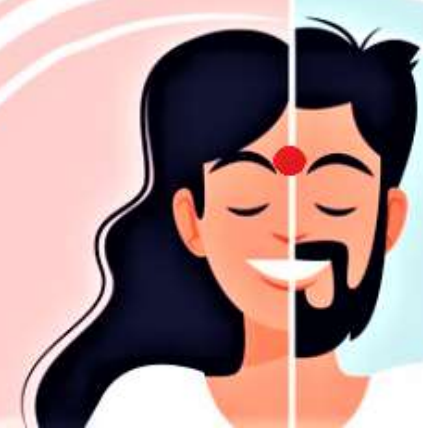

Fig. 2. Woman and Man's Face. 
The Fig. 2 depicts a combination of Female and Male on either side. As it can be seen that Fig. 2 consists of the dot on the forehead therefore when a patient focuses on the middle dot, the information of the female's face will go to the right hemisphere and male's face will go to the left hemisphere. When a patient with a split brain is instructed to point to the picture of faces depicted, the patient will give information which went to his or her RIGHT hemisphere which is generally female. Now, if the patient is asked to tell whether the picture was a male or a female, then he or she would say that it was of a man. Hence, depending on what the patient is needed to try and do, either the right or left hemisphere can dominate [9]. According to prior research, it can be found that Brain consists of two hemispheres: The Left Brain and The Right Brain.

Now relating the existing psychological studies with Artificial Intelligence and Machine Learning techniques and collecting data sets from various psychological tests of various age groups. It is being observed that with time, the brain dominance of a human being changes from left dominance to right or vice-versa.

\subsection{Psychogenic Fever}

Researches have shown that young women suffering from higher levels of anxiety or depression are likely to be exposed to psychogenic fever: There are patients whose high temperature is associated with psychological stress for those who experience episodic or chronic high core body temperature without any inflammatory triggers [10].

\section{Methodology}

\subsection{Data categorization into variables}

1. Age
a) $10-25$
b) $26-40$
c) 40 above

2. Gender
a) male
b) female

3. Brain Dominance

a) Left brain $>$ Right brain

b) Right brain $>$ Left brain

c) left brain = right brain

Firstly a study of vivid research papers and surgeries were conducted on Brain Hemispheres originating from the 1960's till date [11-15]. The study is analyzed based on above mentioned criteria. Data was collected through Google form during the $2^{\text {nd }}$ wave of COVID-19 from various aspects:
* Occupations
* Gender
* Age
* Brain dominance
* Color of the shoe
* Color of the dress

The excel sheet obtained from Google form is converted to CSV (comma separated values) file format. Then variables mentioned above are normalized accordingly for exploratory data analysis. Further, various graphs are plotted with respect to the variables declared which are 
Dendrogram [Fig. 3], Line graphs [Figs. 4, 5], 3-D scatter plot [Fig. 6] and Bar graph [Fig. 7]. Analysis was drawn through the produced graphs, creating relationships among the variables.

\section{Result}

The result says that out of one thousand people, 500 were Female and 500 Male. Out of 500 females, 200 females were found to be right brained and remaining were either $50 \%$ or left brained whereas among 500 males, only 100 were found to be right brained (Table 1).

Table 1. Gender Categorization (Right).

\begin{tabular}{|l|l|l|}
\hline BRAIN/GENDER & MALE (500) & FEMALE(500) \\
\hline RIGHT $>$ LEFT & 100 & 200 \\
\hline LEFT $>$ RIGHT & 330 & 140 \\
\hline $50 \%$ EACH & 70 & 160 \\
\hline
\end{tabular}

Taking the equal number of men and women from the dataset, acquired from the survey conducted during pandemic situation (Lockdown period), some conclusions have been drawn. Using the dataset, the dendrogram plot [Fig. 3] represents that among the dominant Right brained people, Number of Female is more than Male. This outcome shows that a Female is more likely to get depressed or suffer from a mental illness than a Male. Since the dominance of the Right Hemisphere increases the vulnerability towards mental issues.

Fig. 3. Dendrogram 


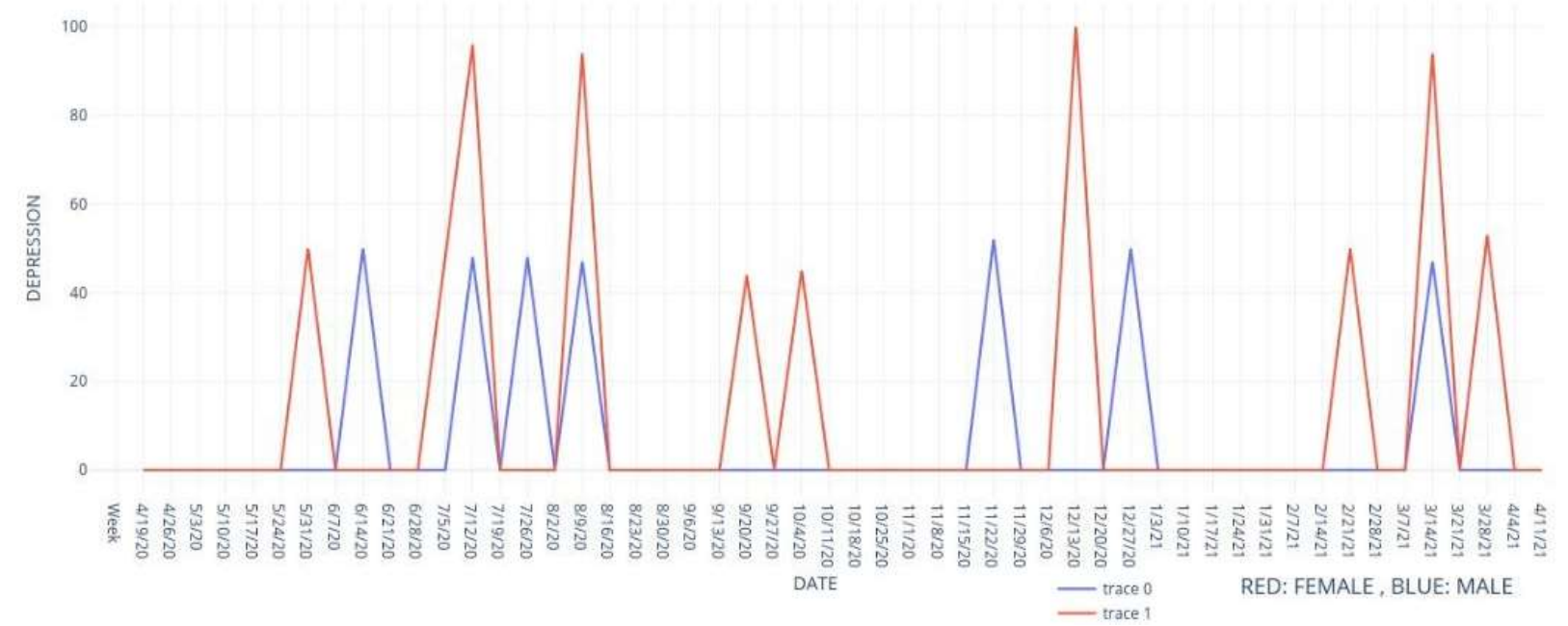

Fig.4. Line Graph for Depression rate.

According to this line graph (India for past 12 months), the Depression rate of Female (red) is always either equal to or greater than Male (blue). The graphs plotted [Fig. 4] support the fact that Female is prone to depression on a higher rate and are mostly right brained as compared to Male. Being a dominant right brainer causes a person to be more creative and imaginative.

MINT AND GRAY

Blue and Grey

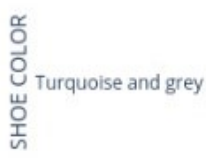

Skyish green

Fig. 5. Line Graph for shoe colors identify by Female and Male.

On analyzing the dataset of people identifying the color of a shoe, the graph [Fig. 5] shows that Female identify multiple color schemes as compared to Male. Male could see the shoe as green and gray only, whereas Female could see other possible combinations (right y axis). 


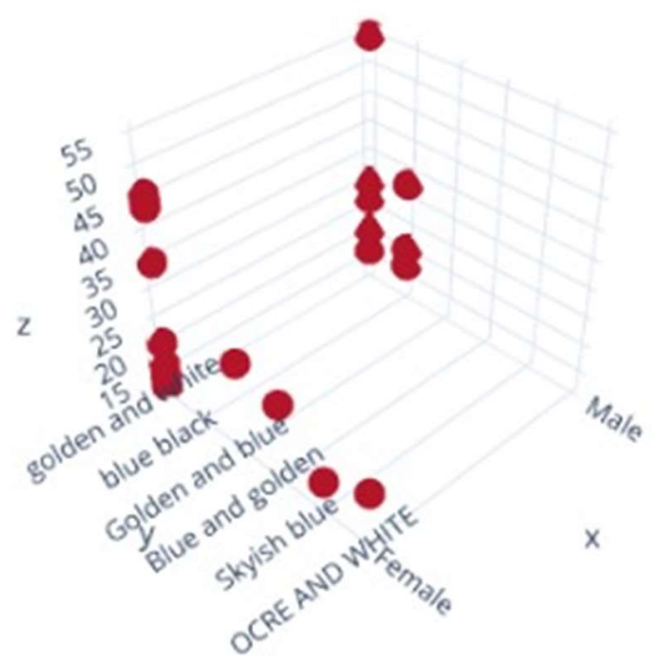

Fig. 6. 3-D Scatter Plot

This 3D scatter plot [Fig. 6] derived from the data set compares three dimensions: Gender ( $\mathrm{x}$ axis), Color of Dress (y axis) and Age (z axis). It represents that females identify more color patterns as compared to Male that lie in the Age group of 10 to 50 .
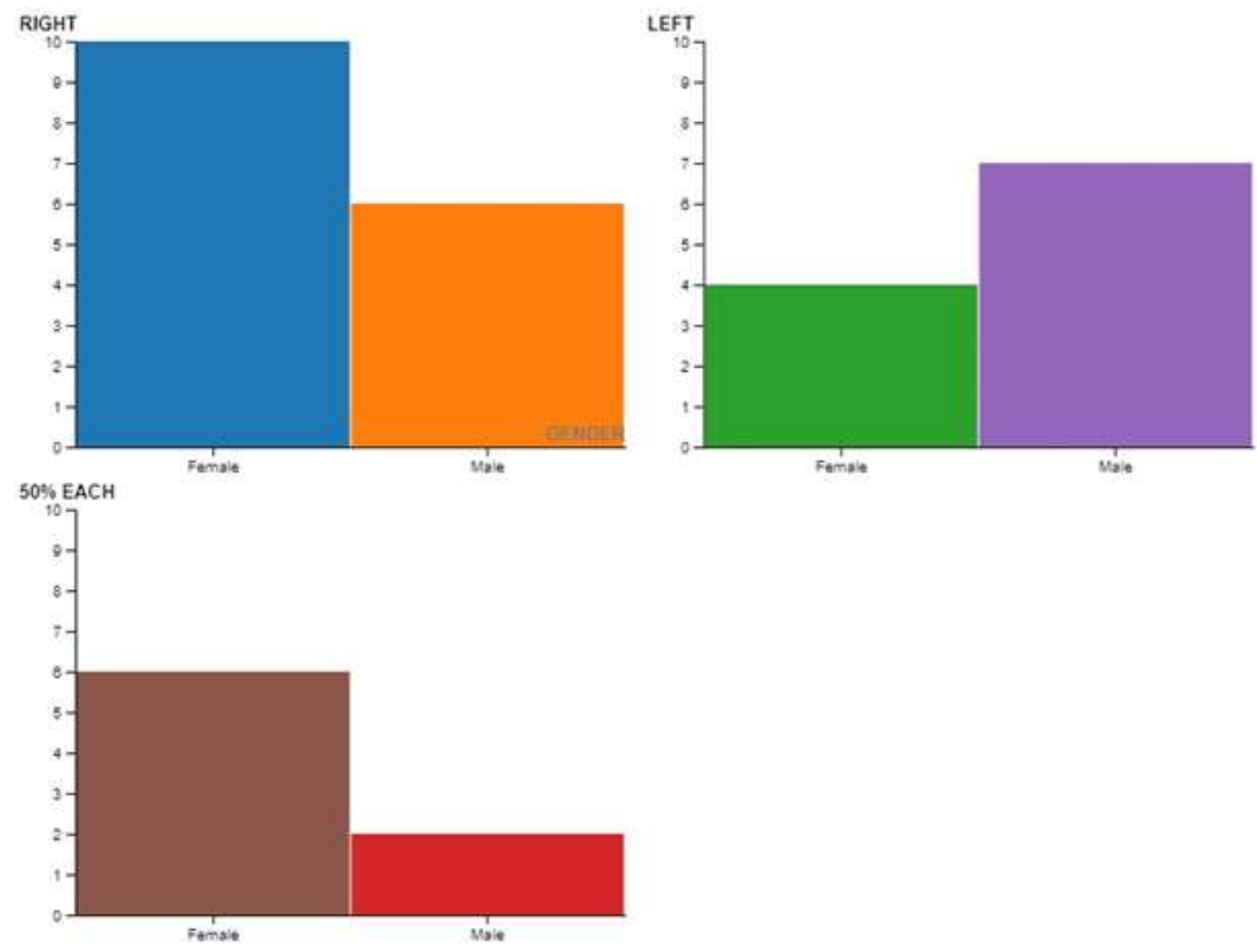

Fig.7. Bar chart 
This Bar Chart [Fig. 7] represents the Dominance of Brain (y axis) with respect to Gender and serializes the data for the age group of 10 to 25 . Here also, It can be inferred that Right Brained and $50 \%$ equal dominance is on a large extent represented by Females. However, Men are Left brain dominating.

\section{Conclusion and Significance}

Working on this research, a dynamic classifier is built. On determining the dominance of the Right Hemisphere, the classifier will help in creating awareness among the viewers regarding the scientific reasons behind such differences. The classifier will suggest interesting games, activities, real time updates accordingly and generate information which explains the phenomenon of Brain Dominance and consequences, whether it may be Left or equal dominance. This will help in dealing with the stressful situation of COVID-19 furthermore accompanied with regular medications and physical exercise. However, it should be kept in mind that $\mathrm{C}$ Chiron, et al. [16] claim that right brain dominance enables children at the age of 3 or 4 . On the contrary, left brain features actives when children are about 7 years old and solely Right Brain is not the only contributor towards mental illness, it requires various other aspects.

- Determining the current status (COVID-19) of one's mind in the early stages will increase the chances of a person avoiding harsh mental issues.

- Discussing such critical matters on a global level helps in reducing the social stigma associated with the mental ailments thus leading to a much worse situation.

- Before cure, the reaction and dealing towards any problem marks a large significance hence, thinking of a mental illness not more than any physical one must be the first step towards a great challenge.

- Especially observing the age group of 10-25 which covers (40-50\%) of the population in India needs more attention peculiarly during COVID-19 pandemic, therefore a new platform which educates as well as gives opportunity to amend the differences will be of great help.

- Discussing lateralization on such a platform will greatly enhance our appreciation

\section{Declaration:} towards different aspects and its effects.

- The authors declare that they have no conflict of interest.

- This research did not receive any specific grant from funding agencies in the public, commercial, or not-for-profit sectors.

- All graphs/figures are self-generated; no copyright is required.

\section{References}

[1] Don Lincoln, Solving the Mystery of the Left-Brain and Right-Brain, Fermi National Accelerator Laboratory (Fermilab). August 26, 2020; From the Lecture Series: Understanding the Misconceptions of Science. https://www.thegreatcoursesdaily.com/solving-the-mystery-ofthe-left-brain-and-right-brain-myth/.

[2] Williams L. M., Defining biotypes for depression and anxiety based on large-scale circuit dysfunction: a theoretical review of the evidence and future directions for clinical translation, Depress Anxiety. 34 (2017) 9-24. https://doi.org/10.1002/da.22556.

[3] Corballis M. C., Left Brain, Right Brain: Facts and Fantasies, PLOS Biology. 12 (2014) 1-6. https://doi.org/10.1371/journal.pbio.1001767. 
[4] Difference between Left Brain and Right Brain. www.mentalup.co/blog/left-brain-rightbrain-dominant. 06 March 2020, Lauran Cole.

[5] Neuroscience for Kids One Brain...or Two? https://faculty.washington.edu/chudler/split.html Copyright (C) 1996-2020, Eric H. Chudler.

[6] Gazzaniga, Michael S., The Split Brain in Man, Scientific American. 217(1967) 24 29. www.jstor.org/stable/24926082.

[7] Roger Sperry, The Split Brain Experiments, Nobelprize.org.

https://www.nobelprize.org/educational/medicine/split-brain/background.html. Nobel Prize Outreach AB 2021. 29 April 2021.

[8] Sperry, Roger W., Cerebral Organization and Behavior, Science. 133 (1961) 1749-1757. https://doi.org/10.1126/science.133.3466.1749.

[9] MRI (Magnetic Resonance Imaging). http://pshs.psd202.org/documents/eforsber/1505061699.pdf.

[10] Oka, Takakazu, Psychogenic fever: how psychological stress affects body temperature in the clinical population, Temperature. 3 (2015) 368-378. https://doi.org/10.1080/23328940.2015.1056907.

[11] Vadim S. Rotenberg, The peculiarity of the right-hemisphere function in depression: solving the paradoxes, Progress in Neuro-Psychopharmacology and Biological Psychiatry. 28 (2004) 113. https://doi.org/10.1016/S0278-5846(03)00163-5.

[12] Hagerty, Sarah L., and Leanne M. Williams, The impact of COVID-19 on mental health: The interactive roles of brain biotypes and human connection, Brain Behavior \& ImmunityHealth. 5 (2020). https://doi.org/10.1016/j.bbih.2020.100078.

[13] Nielsen J. A., Zielinski B. A., Ferguson M. A., Lainhart J. E., Anderson J. S., An Evaluation of the Left-Brain vs. Right-Brain Hypothesis with Resting State Functional Connectivity Magnetic Resonance Imaging, PLOS ONE. 8 (2013) 1-11. https://doi.org/10.1371/journal.pone.0071275.

[14] Sperry, Roger W. Split-brain Approach to Learning Problems. In The Neurosciences: A Study Program (Eds.), Gardner C. Quarton TheodoreMelnechuk, and Francis O. Schmitt, New York: Rockefeller University Press, $1967 . \quad$ pp. 714722. http://people.uncw.edu/puente/sperry/sperrypapers/60s/130-1967.pdf.

[15] Roger W. Sperry, Inside the Brain: The Last Great Frontier, Left-Brain, Right-Brain, The Saturday Review. New York, 9 August, 1975, pp. 30-33. https://www.unz.com/print/SaturdayRev-1975aug09-00030/.

[16] C Chiron, I Jambaque, R Nabbout, R Lounes, A Syrota, O Dulac, The right brain hemisphere is dominant in human infants, Brain. 120 (1997) 10571065. https://doi.org/10.1093/brain/120.6.1057. 